Review

\title{
Optimizing the Immune System to Achieve Control of the Metastatic Malignant Lesion
}

\author{
Myron Arlen ${ }^{1,2} \bowtie$, Philip Arlen ${ }^{2}$ \\ 1. Division of Surgical Oncology, North Shore University Hospital, Manhasset NY, USA \\ 2. Precision Biologics, Great Neck NY, USA
}

$\bowtie$ Corresponding author: myronarlen@yahoo.com

(c) Ivyspring International Publisher. This is an open-access article distributed under the terms of the Creative Commons License (http://creativecommons.org/ licenses/by-nc-nd/3.0/). Reproduction is permitted for personal, noncommercial use, provided that the article is in whole, unmodified, and properly cited.

Received: 2013.04.27; Accepted: 2013.06.10; Published: 2013.06.28

\begin{abstract}
In a recent issue of Nature, an article appeared discussing the issue of "Sizing up a slow assault on Cancer" (Nature 2013;496:14-15). This article attempted to clarify various approaches that the clinician might employ in bringing cancer under control. It also discussed the role of the immune system with regard to its capability for controlling tumor growth. In addition, it covered possible directions that might be taken to improve present responses to immunotherapy based on utilizing T-cell activity directed against the tumor. While there is some validity to the concept that cell mediated immunity is utilized by the host in its attempt to control evolving malignancy, this process actually represents only a minor role taken by the hosts immune system to accomplish what is needed for tumor control. Clinical studies at Precision Biologics have demonstrated that for tumor growth to be effected properly by the hosts immune system, expression of a specific humoral lgGI response directed against immunogenic tumor glycoproteins on the cell surface membrane, constitutes the primary method needed for tumor control. Failure to obtain significant levels of the needed IgG response almost invariably results in recurrence and progression of disease.
\end{abstract}

Key words: IgG response, tumor control

\section{The Host Immune Response}

When fully activated, the more potent part of the host immune mechanism comes into play, especially when the proper immunogen characterizing a specific antigen expressed by the tumor is delivered at a therapeutic level. Mounting evidence from both in-vitro and in-vivo studies at Precision Biologics and other research groups suggest that this process is represented mainly through a $\mathrm{B}$ cell response, producing the needed levels of the specific IgG1 that can bring tumor growth under control (2-5). Under normal circumstances however, the needed level of tumor antigen expression to induce the proper host response is low. Therefore the host attempt to gain control of the situation by the process of tumor surveillance is not an effective mechanism in most circumstances, and progression of disease will be noted.
There is no question that the presences of cytotoxic T-cell lymphocytes permeating a neoplastic process have some relevance in helping to achieve a beneficial response. In a recent study from Sloan Kettering however, in evaluating the presence of TIL (tumor Infiltrating Lymphocytes) cells in patients with colorectal carcinoma undergoing surgery for liver metastasis, it was found that the existence of cytoxic cells could be shown to have some benefit, but the presence of T- regulatory FoxP3 CD8 cells had a negative outcome in terms of survival (6). Similarly, Facciabene et al. Found that T-regs are potent immunosuppressive cells that help to enhance progression of the malignant lesion thru limiting host immunity and promoting tumor angiogenesis (7).

Data that is now being accumulated from various clinical trials have failed to support present 
methods being employed or planned, for delivering cytotoxic cells as a definitive approach to controlling both primary and metastatic lesions. Ongoing clinical trials utilizing targeted monoclonal IgG1's directed against immunogenic tumor proteins now appears to offer the best opportunity for controlling if not curing the metastatic malignant process when the naked antibody is delivered in combination with other antitumor agents. The major goal for employing this approach is first to have isolated and characterized those immunogenic proteins characterizing the malignancy and subsequently for developing the monoclonal capable of targeting the immunogen which most often represents a mutated or post translational modification of an existing oncofetal protein.

\section{The immune response to therapy and the role of cytotoxic $T$ cells}

The term tumor infiltrating lymphocytes (TIL) has been applied to those cells derived from the tumor parenchyma and is believed to represent a host response aimed at helping to control tumor growth. One of the first descriptions characterizing this process was introduced in 1949 when Moore (8) published his classic paper describing tumor infiltrating immunocytes, associated with breast cancer. The process defined by the pathologist, was termed medullary cancer of the breast. Here, patients with this form of disease were considered fortunate in having a better prognosis with regard to survival based on the presence of the lymphocyte infiltrate.

Among several of the first group of patients with breast cancer that we had treated at Sloan-Kettering, a pre surgical biopsy proved the lesion to be medullary carcinoma. This took place several years after Moore published his findings with this form of breast cancer and as such the initial impression was that these patients would have, with little question, a favorable outcome in terms of prognosis. Within months of surgical resection, each of these node negative patients presented with distant metastasis.

In attempting to resolve this issue of host protection by an infiltrative lymphocytic process within the tumor, new questions arose, all with regard to the failure to define an improved survival especially in tumors such as medullary carcinoma. It appeared that much of the information describing prognosis for this tumor type was frequently misleading and that medullary cancer of the breast was in reality a high grade malignancy with poor prognosis. Flores, Arlen et al. (9) reviewed over 100 cases of this so called disease process, dividing the patients into 4 groups based on the level of lymphocyte infiltration It became apparent early on, that the greater the level of lymphocyte infiltration within the breast tissue, the poorer the prognosis. It was very obvious that the presence of tumor infiltrating lymphocytes did not bode well in terms of controlling the malignant process and did not, as originally described, represent a major attempt by the host to control tumor as it continued to grow.

This particular finding continued to remain equivocal until Dudley and Rosenberg (10), in isolating and culturing infiltrating lymphocytes from tumor specimens, now termed TILL cells, was able to demonstrate some benefit, but still an insignificant response following the isolation, scaling up, and delivery of large numbers of such TIL cells to the host presenting with metastatic cancer. While a partial response was seen in a few patients with metastatic melanoma, it did not appear to represent, from a clinical point of view, a major change in altering survival of those patients with metastatic disease. In 2006 (11) Morgan and Dudley further demonstrated that the receptor of T-cells could be transferred to immune cells as a form of gene therapy and again when delivered in combination with chemotherapy and high dose IL-2, they could demonstrate an occasional beneficial response. The problem that arose however was that in a similar situation, some patients could be shown to have a partial response independent of the use of TIL cells, when IL-2 was given along with chemotherapy. A number of ongoing studies are still being devised and utilized whereby activated $\mathrm{T}$ cells are being employed in melanoma studies. Whether any major benefit will be obtained from the data accrued in these studies is controversial.

Jamieson has looked into the possibility that infiltrating lymphocytes representing the presence of a high grade inflammatory response in patients with pancreatic cancer does confer some beneficial response. It appears in some instances that the presence of this response does suggest prolongation in survival. Whether any specific cell type such as the presence of NK cells is associated with clinical benefit was not apparent (12).

A further analysis of the possible mechanisms resulting from activation of a cellular immune response appeared in a study by Schietinger et al.(13) They reported that bystander killing of cancer required the cooperation of $\mathrm{CD}^{+}$and $\mathrm{CD}^{+}$cells. Experimental cancers could be eradicated by such $\mathrm{T}$ cell combination targeting the tumor stroma as a major factor in bringing the lesion under control.

Jiang and Mann (14) looked further into the phenomenon and possible benefit of immune cells entering into the tumor matrix. They described the activity of such infiltrating cells with regard to tumor lysis and noted rather, that lymphocytes entering 
tumor parenchyma appeared in many instances to help chaperone tumor cells within the lesion to the lymphatic and vascular systems, thus enabling the tumor cells to metastasize. No antitumor response was noted by these cells in their association with adjacent malignant cells. They therefore categorized infiltration of tumor by lymphocytes in negative terms and regarded this phenomenon as a failure in the host response, attempting to bring existing tumor under control.

Where it was felt that existing immunocytes in the host demonstrated some degree of impaired function, specific monoclonals were developed that could be used to restore these functions (15). As such Immunomodulatory antibodies directed against cytoxic $\mathrm{T}$ cell associated antigen 4 (CTLA-4/CD52) and programmed cell death ligand 1 (PDL-1/CD274) were developed and shown to demonstrate evidence of improvement in clinical response. There have however been a limited number of patients who appear to benefit from such immunomodulatory antibodies. The factors involved here were felt to be possibly being due to an inadequate number of, as well as impaired cytotoxic lymphocyte activity. With this in mind, a more plausible approach appeared to be in directing the immune system to target those immunogenic proteins found on the surface of the tumor; those that characterize the specific tumor system, i.e., colon, pancreas, lung etc.

While most foreign cells considered as invaders, such as bacteria and viruses, contain sufficient levels of the target antigen to allow the host immune system to control their presence, when one considers the cancer cell as a potential foreign invader, the level of tumor antigen/immunogen present is too low to allow for effective control. Here one needs to define the tumor associated antigen (TAA) and present it to the host in sufficient amounts (threshold level) to allow an effective host immune response to occur.

\section{Clinical use of Tumor Associated Antigen and the Resulting Immune Response}

A more rational approach leading to an understanding of how host immunity could be properly as well as effectively activated was described by Hollinshead (16). During the 1980's when the Hollinshead vaccine was approved for use in treating patients undergoing surgery for advanced neoplastic lesions, the length of survival among those receiving pooled allogeneic antigen preparations was found to more than double over those undergoing surgery alone Whereas recipients receiving the vaccine developed enhancement in both their cell and humoral responses, the one factor that appeared most responsible for improved survival, was the ability to produce an adequate and prolonged level of an IgG1 antibody directed against the tumor antigen. Failure to develop a sustained serum level of the antibody capable of targeting tumor antigen expressed on the cell surface membrane, resulted in recurrence in spite of the presence of an adequate $\mathrm{T}$ cell response. The important factor here was that the vaccine was specific for the tumor system being treated and that it was delivered at a threshold level of approximately $700-1000 \mu \mathrm{gm}$. depending on the degree of purity. This preparation was given at antigenic levels more than 20 times that of the immunogenic proteins present within the patient's own tumor system. This in essence precluded the use of the patients own tumor for developing effective personalized vaccines as had been directed by the FDA.

In a paper by Hollinshead, Takita and Stewart (17), one can easily recognize the improved and sustained survival seen in lung cancer patients who received a tumor antigen preparation. Pooled allogeneic protein was delivered in the form of a vaccine plus adjuvant following resection. There were no restrictions in the use of such preparations at the time, since HPV, hepatitis B and C and HIV were not considered major threats in producing and delivering such preparations. Results obtained for patients receivng such vaccines in the above study were compared to those having undergone surgery alone and who therefore served as controls (18) (Fig.1).

On completion of the vaccine trials going into the 1990 's and at the suggestion of the FDA, it was felt that the pooled allogeneic vaccines could have some form of viral contamination and as such should not be further utilized for therapy. Rather they suggested that the tumor proteins in the vaccine be used to produce monoclonal antibodies and that such antibodies be employed to further define the structure of the pooled proteins by immunoprecipitation and mass spectroscopy. At this particular time, ADCC (antibody dependent cell cytotoxicity) was coming into vogue for evaluating the ability of antibodies to induce a form of tumor lysis. Such studies were being performed on carbohydrate antibodies such as 17.1a. As a control, one of our monoclonals targeting colon cancer termed 31.1 was utilized. It is readily apparent that a protein antibody such as 31.1 used to define an immunogenic glycoprotein can be far more effective than what one sees with a carbohydrate antibody such as 17.1a (Fig.2). Considering that colon tumor cells in culture, proliferate at about $6-10 \%$ q $6 \mathrm{hrs}$. One would expect from this data that that nothing less than a $30 \%$ ADCC would give the needed clinical response. When the monoclonal antibody 17.1a, now termed 
Panorex, was utilized in clinical trials for patients with Dukes C2 colon cancer, it was not unexpected to find absence of a significant improvement in survival. One would anticipate that a protein derived monoclonal, with an ADCC in excess of $50 \%$ and delivered at the right therapeutic dose could achieve a long needed clinical response. This became the target of the therapeutic trials at Precision Biologics knowing that with monoclonals delivered intravenously, the rapid tumor response seen in a matter of hours could be an effective way for treating patients with recurrent cancer having failed chemotherapy (19).

Animal studies that were performed were planned to demonstrate that the proper IgG1's deliv- ered to nude mice presenting with fully established human colon or pancreatic carcinoma could be brought under control. In all such studies comparing nonspecific IgGs to those IgG'1 derived from the tumor antigen preparations of Hollinshead, a major antitumor response was seen with the specific monoclonals. The antibodies for potential human trials were chimerized since any ADCC response would rely on the delivery by the antibody, of NK cells attached to receptors on the human Fc. Each of the monoclonals that have been developed by our group for therapeutic use is now in the process of being humanized.

\section{Squamous Malignancies TAA Immunotherapy of Lung Cancer}

Survival afterTAA Immunotherapy

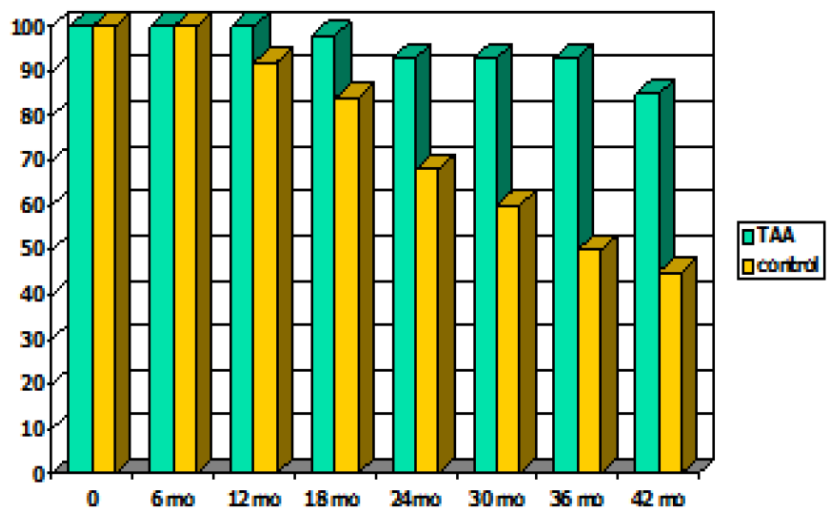

Fig I. Demonstrates a decrease survival that progresses well beyond 42 months in patients failing to receive immunotherapy. Where the survival of the immunized host remained above $75 \%$ at 5 years, the major factor resulting in total control of the tumor with regard to survival appeared to be the ability or failure to produce an IgGI targeting the tumor immunogen.

\section{ADCC with Chimeric Monoclonal NPC2}

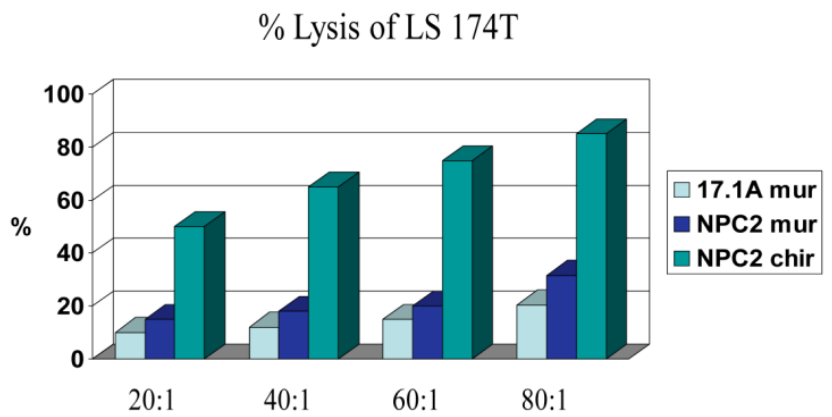

Effector to Tumor Cell Ratio

Fig 2. The ADCC response at an effector to tumor ratio of $80: 1$ produced better than an $75 \%$ ADCC/ when the chimeric version was employed. This response is due to delivery of NK cells to the tumor when the NK cell binds to an NK receptor on the $\mathrm{Fc}$ of the monoclonal requiring that such antibodies if not delivered in the human or humanized form must receive at least a chimeric version. 
Aab-Induced Anti-Tumor Efficacy on Established Tumors

- Chimeric antibody administered after ten days of tumor growth

- Top mouse treated as a control with non-specific antibody

- Bottom mouse treated with the NPC anti-colon cancer-specific $\underline{\mathrm{mAb}}$

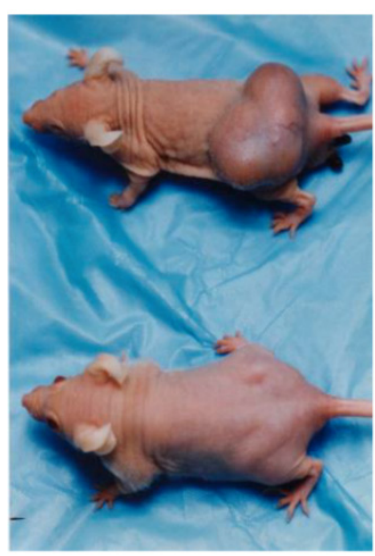

Fig 3. demonstrates the ability of the monoclonal antibody for Colon Cancer targeting the immunogenic tumor antigen for tumor regression. The animal given a non-specific antibody had progression of tumor growth. Antibody was not given along with the human cells that then did or didn't establish tumor growth but was used for treatment only after the large tumor growth became apparent.

\section{Therapeutic use of Monoclonal Antibody Preparations}

In terms of colon and pancreas cancer studies, we are presently in Phase IIa trials with the chimeric version of the antibody Neo 102 targeting an oncofetal protein expressed in both colon and pancreas cancer. Three immunogenic antigens have been defined for these tumors. Each represents an oncofetal status of the expressed protein, wherein at the time of malignant expression of the lesion a mutation has been characterized which represents either a gene mutation or post translational modification within the core peptide of the corresponding oncofetal protein. The monoclonals that target these proteins are effective in both diagnosing the presence of shed antigen representing tumor markers in the serum, are capable of detecting the presence of early as well as late transformation within normal appearing cells, and when delivered intravenously, can hunt, seek and destroy any cell expressing the antigen. No normal tissue has been found to express these tumor antigens. A recent publication by Arlen et al from our group covers how our monoclonals effect the clinical activity of colon and pancreas cancer and also serve to diagnose the presence of such lesions by ELISA and IHC (20).

Considering that the monoclonals we are employing induce a number of different antitumor responses in addition to ADCC and that the effect on tumor can occur within several hrs of administration, it appeared ideal to employ monoclonal antibody therapy in patients with recurrent tumor having failed chemotherapy such as those with metastatic pancreas and colon cancer. We are hoping that as trials progress, that we can show major changes in clinical response when the naked antibody is delivered in combination with chemotherapy and that a possible follow up to the initial antibody approach will be delivery of the antibody conjugated with an alpha emitter and possible addition of immune stimulants to further enhance an overall clinical response.

\section{Conclusion}

While many different approaches have been investigated in terms of controlling cancer growth including use of those monoclonal antibodies that minimize vascular proliferation or attack epidermal growth factors as well as blocking signaling pathways thru tyrosine kinase inhibitors, we believe that the Precision Biologic approach using targeted monoclonal therapy is one of the more effective approaches to utilize. When delivered initially as a naked therapeutic monoclonal antibody and later in combination with other antitumor agents, it is felt that this approach can offer the best possibility for achieving a cure, even in the metastatic setting.

Later on, as we achieve our goals in targeting recurrent tumors with our therapeutic antibodies, the use of peptide vaccines, several which have already been designed, will be introduced to help prevent the 
high rate of recurrence of malignancy seen after complex operations such as the Whipple (pancreatectomy). Initial evaluation of 12-20 mer peptides derived from the epitope binding sites on the altered TAA from which the monoclonals have been derived; suggest that such epitopes when delivered in the form of a peptide vaccine can turn on the full immune response. Further on in the future, we may consider employing polyvalent peptide combinations to possibly prevent the development of a malignant disease process similar to what can achieve by employing antiviral vaccines. All of these approaches are anticipated to bring "cancer" as a disease under control if not eliminate it.

\section{Competing Interests}

The authors have declared that no competing interest exists.

\section{References}

1. Ledford H. Sizing up a Slow Assault on Cancer -editorial. Nature 2013:496:14-15.

2. Hemstreet GP 3rd, Rossi GR, Pisarev VM, Enke CA, et al. Cellular immunotherapy study of prostate cancer patients and resulting IgG responses to peptide epitopes predicted from prostate tumor-associated autoantigens. J Immunother. 2013;36 :57-65.

3. Aarntzen EH, de Vries II, Göertz JH, Beldhuis-Valkis M. Humoral anti-KLH responses in cancer patients treated with dendritic cell-based immunotherapy are dictated by different vaccination parameters. Cancer Immunol Immunother.2012;61:2003-11.

4. Weber JS, Hamid O, Chasalow SD, Wu DY, Parker SM, Galbraith S, Gnjatic S, et al. Ipilimumab Increases activated T-cells and enhances humoral Immunity in with advanced melanoma. J Immunother. 2012; 35: 89-97.

5. Arlen M, Arlen P, Bristol A, Wang X, et al. The Use of Specific Monoclonal Antibodies to target Immunogenic tumor membrane proteins in Patients with Recurrent Pancreatic and Colon Cancer. Current Drug Delivery. 2012; 9:52-56.

6. Katz SC, Bamboat ZM, Maker AV, Shia J, Pillarisetty VG, et al. Regulatory T Cell Infiltration Predicts Outcome Following Resection o Colorectal Liver Metastasis. Ann Surgical Oncology 2013;20:946-9537.

7. Facciabene A, Motz GT, and Coukos T- Rdgulatory Cells: Key Players in Tumor Immune Escape and Angiogenesis. Cancer Research 2012; 72: 2162- 2170 .

8. Moore OS, Foote FW. The relatively favorable prognosis of Medullary Carcinoma of the Breast. Cancer 1949;2:635-642

9. Flores L, Arlen M, Elguezabal A, Livingston S, Levowitz B. Host Tumor Relationships in Medullary Carcinoma of the Breast. Surg Gynecol Obstet 1974; 139:683-8.

10. Dudley ME, Wunderlich JR, et al. Cancer regression and autoimmunity inpatients after clonal repopulation with antitumor lymphocytes. Science 2002; 298: 850-854.

11. Morgan RA, et al. Cancer regression in patients after transfer of genetically engineered lymphocytes. Science 2006; 314: 126-129

12. Jamieson NB, Mohamed M, Oiem KA, et al. The Relationship between Tumor Inflammatory Cell Infiltrate and Outcome in Patients with Pancreatic Ductal Adenocarcinoma. Ann.surg. Oncol. 2012; 19:3581-359013.

13. Schleitenger R, Philips M, Liu RB, et al. Bystander killing of Cancer reuires the cooperation of CD4+ and CD8 T-cells During the Effector Phase. J. Exp. Medicine 2010; 207:1-21.

14. Jiang B, Mason J, Jewett A, Liu Ml, Chen W, Qian J, Ding Y, Ding S, Ni M, Zhang X, Man Yg. Tumor-Infiltrating Immune Cells: Triggers for Tumor Capsule Disruption and Tumor Progression?. Int J Med Sci 2013; 10(5):475-497.

15. Aerts JG, Hegmans JP. Tumor specific Cytotoxic T Cells are Cruciat for Efficacy of Immunomodulatory Antibodies in Patients with Lung Cancer. Cancer Research 2013; 73: 2381-218316.
16. Hollinshead AC. Active specific immunotherapy. In: Higgins L, ed. Immunotherapy of Human Cancer. NY: Raven Press. 1978: 213-233.

17. Hollinshead AC, Takita H, Stewart T, Raman S. Specific Active Lung Cancer Immunotherapy. Cancer 1988; 62: 1662-1671.

18. Takita H, Hollinshead AC, Adler $\mathrm{RH}$, et al. Adjuvant, specific, active immunotherapy for resectable squamous cell lung carcinoma: a 5 year survival analysis. J Surg Oncol. 1991; 46:9-14.

19. Arlen M, Arlen P, Tsang A, Wang XP, Gupta R. The Therapeutic Value of Monoclonal Antibodies Directed against Immunogenic Tumor Glycoproteins. J Cancer 2010; 1:209-220.

20. Arlen M, Wang X, Luka J, et al. The Use of Specific Monoclonal Antibodies to Target Immunogenic Tumor Membrane Proteins in Patinets with Recurrent Pancreatic and Colon Cancer. Current Drug Deliv. 2012; 9: 52-56. 